\title{
Vitamin D Deficiency, Atherogenic Index and Some Demographis Data as Risk Factors with The Prevelance of Diabetic Peripheral Polyneuropathy in Type II Diabetic Females
}

\author{
Abdulrahman M Alhowikan, Fawzia AAl Rouq, Laila M Aldokhi, Hana Alzamil and Faten A Zakareia* \\ Department of physiology, Saudi Arabia \\ *Corresponding author: Faten abulhady zakareia, Professor and consultant of clinical physiology, Department of physiology and \\ clinical physiology, College of medicine, Riyadh, Saudi Arabia
}

\begin{tabular}{l}
\hline ARTICLE INFO \\
\hline Received: 蔧July 29, 2019 \\
Published: 蔧 August 12, 2019 \\
\hline Citation: Abdulrahman M Alhowikan, \\
Fawzia AAl Rouq, Laila M Aldokhi, Hana \\
Alzamil, Faten A Zakareia. Vitamin D \\
Deficiency, Atherogenic Index and Some \\
Demographis Data as Risk Factors with \\
The Prevelance of Diabetic Peripheral \\
Polyneuropathy in Type II Diabetic Fe- \\
males. Biomed J Sci \& Tech Res 20(3)- \\
2019. BJSTR. MS.ID.003460.
\end{tabular}

\section{ABSTRACT}

Objectives: To explore the possible association between vitamin D deficiency, body mass index (BMI), atherogenic index (AI), and glycosylated heamoglobin (HbA1c) with diabetic peripheral neuropathy(DPN). Also, with the electrophysiological measures of nerve impairment and sensory loss

Methods: This study was conducted at the Clinical Physiology Laboratory and Diabetes Research Center of King Abdul Aziz University Hospital (Riyadh, Saudi Arabia) , between January 2018 to May 2019 ,involved 15 subjects with controlled diabetes ,30 diabetic patients with possible DPN and 50 diabetic patient with DPN.Electrophysiological nerve conduction studies and serum 25(OH)D ,lipid profile and HbA1c were measured.

Results: the present study reveals a significant deficiency of Vit D in group III (late DPN) compared to early DPN (group II) and its negative correlation with DPN electrophysiological measures of median, ulnar, peroneal and tibial nerves motor potential latency, and with median and sural nerves sensory potential latency, also its positive correlation with sural nerve sensory amplitude, also with sensory and motor nerves conduction velocity. The current study showed that subjects who had late DPN had a longer duration of diabetes, and higher mean hemoglobin A1c, atherogenic index, BMI than those who have possible early neuropathy. Deficiency of Vit D, high atherogenic index, BMI and HbA1c are predictors and risk factors for DPN.

Conclusion: Vit D deficiency, hyperglycemia, $\mathrm{Hb}$ A1c, obesity and dyslipidemia are independent risk factors of DPN as a microvascular complication in T2DM patients. Sensory large myelinated nerve integrity also correlated with BMI and atherogenic index.

\section{Introduction}

Diabetic Polyneuropathy (DPN) is the most common complication of diabetes mellitus. Diabetic neuropathy causes dysfunction of small and large nerve fibers [1]. Sensorimotor diabetic neuropathy involves both sensory and motor function, where paresthesias occur along with decreased strength in the lower limb muscles [2]. Loss of pain, temperature perception, and a predisposition to foot ulceration are characteristic to smallfiber peripheral neuropathy. Loss of proprioception, deficits in nerve conduction, and weakness of distal limbs occur with large- fiber nerve dysfunctions [3]. Different factors recognized in the pathogenesis of DPN are vascular insufficiency, loss of growth factor trophism, and autoimmune destruction of small unmyelinated nerves (C fibers) [4]. The abnormal vasomotor responses may be also related to insulin resistance, hyperinsulinemia, hyperglycemia, endothelial dysfunction and dyslipidemia. Atherosclerotic changes in Type 2 diabetes are characterized by abnormal vascular function, which may ultimately contribute to the clinical manifestations of neuropathy, micro- and macrovascular disease [5]. 
Hyperglycemia may affect the peripheral sensory nerves through several mechanisms to induce diabetic neuropathy. The increased intracellular sorbitol accumulation decreased neuronal blood flow, and peripheral nerve hypoxia in hyperglycemia can cause direct neuronal damage [5]. The auto-oxidation of glucose induces endothelial damage through increased production of Reactive Oxygen Species (ROS), and advanced glycation end products. induces vasoconstriction and reduces neuronal blood flow by activation of protein kinase C [6]. Vitamin D is an essential hormone obtained from the diet or skin synthesis. It can be hydroxylated in the liver into 25-hydroxyvitamin D (25[OH]D), then converted to its active form by 1 - hydroxylation in the renal proximal tubules into 1,25 dihydroxyvitamin D $(1,25[\mathrm{OH}] \mathrm{D})$ [7]. Studies have confirmed the occurrence of low serum 25(OH)D levels in patients with chronic kidney disease [7]. Vitamin D has a classical function in maintaining calcium and phosphate homeostasis, but also it has a potential role in maintaining immunity, vascular function, cardiomyocyte health, inflammation, insulin resistance and in lowering the urinary albumin concentration in patients with chronic kidney disease [8].

Celil et al. [9] have been stated that Vitamin D is a known suppressor of renin biosynthesis, and vitamin D deficiency has been associated with progression of Chronic Kidney Disease (CKD). Patients with type 2 diabetes and CKD have an exceptionally high rate of severe $25-\mathrm{OH}$ vitamin D deficiency. Low vitamin D levels are suggested to be associated with different impaired glucose metabolism states including diabetes mellitus. Vitamin D deficiency is supposedly higher in type 2 diabetic patients compared to healthy individuals [10]. Vitamin D deficiency contributes to the etiology and progression of type 2 diabetes. 25- $\mathrm{OH}$ vitamin D concentrations were found to be lower in patients with type 2 diabetes with impaired glucose tolerance than in controls [10]. Diabetic nephropathy is a major microvascular complication of diabetes mellitus [11]. Animal studies have shown that calcitriol supplement can reduce albuminuria in mice with diabetic nephropathy [11].

The relation of Vit D with diabetes complications is poorly studied in Saudi Arabia and needs to be more illucidated.

Dyslipidemia describes as elevated plasma concentration of lipid (Triglyceride (TG) and Total Cholesterol (TC) and their blood transporting lipoproteins (HDL- Cholesterol, LDL-Cholesterol, VLDL-Cholesterol). There is a strong association between incidence of CVD and high level of LDL-C and also low level of HDL-C ,therefore the LDL-C/ HDL-C ratio is often calculated to estimate cardiovascular risk as indicated by strong scientific evidence .On the other hand, high level of TG has been related with an increased LDL-C particles and increased cardiovascular risk [12]. On that basis, atherogenic dyslipidemia, defined as high LDL-C/HDL-C ratio and hyper TG, is associated with high cardiovascular risk [12]. Atherogenic Index (AI) is inversily related and significantly correlated with insulin sensitivity, and it has been reported that $\mathrm{AI}$ correlates with insulin resistance (HOMA- IR) [13]. But AI relation to DPN needs more investigations to be clarified and highlighted. To the best of our knowledge, the correlation of vitamin D deficiency as a risk factor in patients with diabetic peripheral neuropathy is rarely investigated in Saudi diabetic patients, so it remains unclear and needs to be illucidated. The goal of the present study is to evaluate a possible association between vitamin D deficiency and DPN. Also the current study aimed to determine the correlation of as Body Mass Index (BMI), Total Cholesterol (TC), High Density Lipoproteins (HDL), Low Density Lipoproteins (LDL), Atherogenic Index(AI) as possible risk factors for DPN , and to correlate vitamin D levels and these risk factors with the electrophysiological measures of nerve impairment and sensory loss which are the gold standard for determining motor and sensory nerve function in DPN.

\section{Methods}

This observational cross-sectional study was conducted at the Clinical Physiology Laboratory and Diabetes Research Center of King Abdul Aziz University Hospital (Riyadh, Saudi Arabia) , and was conducted between January 2018 to May 2019, This study was approved by the Ethics Committee of King Khalid University Hospital, and all of the procedures were performed in accordance with ethical approval institutional guidelines. The study protocol followed the ethical guidelines of the most recent Declaration of Helsinki. All biochemical parameters were measured in a biochemistry laboratory at the biochemistry laboratory of King Khalid and King Abdulaziz University Hospital.

\section{Subjects}

Subjects with Type 2 diabetes who had neuropathy symptoms, or symptoms consistent with distal symmetric polyneuropathy in the opinion of the investigators were recruited. Those with $>5$ years of symptoms were mainly recruited. A consecutive sampling method was used. A provisional list of patients eligible to participate in the study was made from the records. On clinic days, all eligible patients were approached and asked to participate in the study. Those who accepted to participate signed the informed consent after reading the patient information leaflet.

Patients who met and fulfill the following inclusion criteria were eligible to participate:

a. Willingness to participate in the study by signing the informed consent

b. Diagnosed Type 2 diabetic patients. Metabolic control was assessed on the basis of Glycosylated HbA1c level. They were on oral hypoglycemics. They had normal blood pressure and kidney function.

c. Patients were examined for the presence of DPN.

d. Patients were excluded from the study if one or more of the following exclusion criteria was present:

e. Patients with Type 1 diabetes, diagnosed by history and clinical examination 
f. Diabetic nephropathy or renal failure. Urinary albumin excretion 30-300 mg/24 h Was regarded as microalbuminuria.

g. Patients with diabetic retinopathy, diagnosed by standard ophthalmological evaluation to identify nonproliferative or proliferative diabetic retinopathy.

h. Patients take any vit D supplementation.

i. Hypothyroidism or hyperthyroidism, diagnosed by abnormal thyroid function tests

j. Presence of coronary artery disease or congestive heart failure, diagnosed by ECG

k. Pregnancy and lactating women

l. The diabetic patients with liver dysfunction, concurrent illnesses, smokers and participated in other clinical studies within the last 3 months.

m. Patients with any psychological or neurological disorder.

\section{Variables and Data Collection}

In this cross-sectional study a total of 150 eligible outpatient who had initially agreed to participate were enrolled and identified, they were diabetic females attending the Diabetes Research Centre of King Abdul Aziz University Hospital(Riyadh, Saudi Arabia) ,diagnosed with type 2 diabetes mellitus for the last 10 years and on metformin or sulfonylurea or both as stable treatment for the last 3 months .Patients were in the same age group of 35-60 years, they were subjected to history taking, thorough clinical examination , and laboratory investigations to establish diagnosis of type 2 diabetes and DPN, they were diagnosed according to American Diabetes Association standards. Twenty did not agree to participate in this study and the remained 130 patients signed the informed consent. Of those who signed, 35 patients were excluded from the study as they have other diabetic complications as mentioned in the exclusion section, 20 had prolipherative retinopathy and 15 had nephropathy as diagnosed by the laboratory investigations and fundus ophthalmoscopy.

The final analysis was performed based on data obtained from the remaining 95 participants. Prior to the start of the study, they received information about the study and its aims and gave their informed consent to participate. Peripheral neuropathy (DPN) was defined based on certain criteria: symptoms, signs abnormal NCS (defined as abnormal median, Sural or Peroneal motor or sensory amplitude or motor and sensory conduction velocity using age adjusted normative data). subjects who fulfilled 0 criteria were classified as having no neuropathy. We found 5 patients has no signs and symptoms of DPN or other criteria of DPN according to electrophysiological findings, they were included as a control group. In this study DPN was defined according to the presence of peripheral sensorimotor neuropathy symptoms plus abnormal nerve conduction in at least 2 peripheral nerves.
Those fulfilling 1-2 criteria were defined as early possible neuropathy (they have either only signs\& symptoms or have signs\& symptoms plus abnormal nerve conduction in only one peripheral nerve), they were 30 patients. Those fulfilling three or more (e.g. symptoms and signs in addition to one confirmatory test, symptoms or signs in addition to two or more confirmatory tests, or "laboratory based" diagnosis based on three confirmatory tests without signs or symptoms). Patients with clinical neuropathy were diagnosed on the basis of: abnormal electrodiagnostic tests with decreased nerve conduction velocity (NCV), or decreased amplitudes, or prolonged sensory or motor distal latencies, and abnormal quantitative sensory tests for vibration, tactile, thermal warming, and cooling thresholds. They were defined as patients with DPN with neuropathy and were 50 patients.

The patients were divided into three main test groups: -

a) Group I: - Includes 15 diabetic patients without DPN or other diabetic complications

b) Group II: - Includes 30 diabetic patients had early DPN.

c) Group III: - Includes 50 diabetic patients had late DPN.

n. Demographic characteristics: -Demographic characteristics of the enrolled patients were recorded as age, duration of diabetes, weight and height were measured and Body Mass Index (BMI) of each subject was calculated by dividing weight by the square of height $\left(\mathrm{Kg} / \mathrm{m}^{2}\right)$.

\section{Biochemical Investigations}

Sample Collection: All diabetic individuals fasted for 8 hours and $10 \mathrm{ml}$ of their venous blood was collected in EDTA tubes, centrifuged at $3500 \mathrm{rpm}$ for $10 \mathrm{~min}$ for plasma collection and stored at $-70^{\circ} \mathrm{C}$. Plasma analysis was done to measure the standard range of biochemical parameters that would be tested for in patients with diabetes mellitus, using standard methods. The Fasting Plasma Glucose (FPG) level was measured by a hexokinase assay (Olympus Diagnostics, Tokyo, Japan). For glycosylated Haemoglobin (HbA1c) analysis, (5 ml blood) were collected into tubes containing separation gel coagulant or ethylenediaminetetraacetic acid and then centrifuged at room temperature for $5 \mathrm{~min}$ and stored at -20 C prior to use. Then it was assessed by high-pressure liquid chromatography (Trinity Biotech, Premier Hb9210, Ireland). The low-density lipoprotein cholesterol (LDL-C), fasting serum total cholesterol (TC), triglyceride (TG) and high-density lipoprotein cholesterol (HDL-C) were measured by an enzymatic assay (Wako Diagnostics, Tokyo, Japan). Then the plasma Atherogenic index was calculated as log (TG/HDL-C), or LDL-C/ HDL-C. All participants underwent a $24 \mathrm{~h}$ urinary albumin excretion test by measuring urinary albumin ( $\mathrm{N}$ antiserum to human albumin kit; BN prospec, Siemens, Germany) and urinary creatinine (Creatinine Plus version 2 kit; Cobas c701, Roche,Germany) according to the manufacturer's instructions to exclude diabetic nephropathy also they underwent fundus examination to exclude diabetic retinopathy. 
Serum levels of Vitamin D: In the present study, 25(OH)D was used as a sensitive marker of total body vitamin D storage. Five ml of blood was collected for analysis of serum 25-hydroxy Vitamin D (25-OH Vitamin D) by using high-pressure liquid chromatography, as described previously.(14) 25-hydroxyvitamin D level of 25-80 $\mathrm{ng} / \mathrm{ml}$ is considered normal, $10-24 \mathrm{ng} / \mathrm{ml}$ is considered mild to moderate deficiency but levels less than $10 \mathrm{ng} / \mathrm{ml}$ is considered severe vitamin D deficiency [14].

Quantitative Sensory and Motor Tests for DPN: All groups were compared for presence of DPN in peripheral nerves (motor \& sensory). Concerning the procedures for determining the diagnosis of DPN, it was assessed by consultants of clinical physiology by clinical examination and measurement of NCSs. Nerve conduction studies (NCS) included bilateral sural , ulnar and median sensory amplitude, latency and conduction velocity a measure of large sensory fibers integrety, and for large myelinated fiber integrity we measured median, ulnar, tibial and peroneal motor amplitude, distal latency and conduction velocity. We also examined sensory impairment by quantitative assessment of thermal sensitivity and other neurological examinations. A questionnaire on symptoms of peripheral neuropathy was completed by all patients, and by all control subjects. The tendon reflexes in the quadriceps, gastrocnemius, and biceps muscles were classified, either as present or absent, for the sensations of light touch, pain (pin prick), temperature, and joint position in the index finger and big toe bilaterally were carried out.

All patients in group II and III had peripheral neurologicalsymptoms (tingling or numbness). Some of the patients had loss of sense of light touch, pain, or joint position, and 24 patients had abnormal vibration sense, whereas 6 patients had normal vibration sense. The nerve conduction tests were performed as described by previous reports [15] in the Department of Clinical Physiology with standard surface recording techniques using an electromyography type Spirit Nicolet Viking (Nicolet- Biomedical Inc, Madison, WI, USA). Unilateral sensory NCS were performed antidromically on median and sural nerves, motor nerve conduction studies (NCS) were performed on the median, ulnar, tibial and peroneal nerves. For motor nerves, the electromyographic settings of the machine were: frequency (8 Hertz [Hz]-8 kiloHertz [kHz]), sweep speed (5 $\mathrm{msec} /$ division), gain $(1000 \mathrm{uV})$, stimulation intensity (400 V), and duration $(0.1 \mathrm{msec})$. While the settings for sensory nerves were frequency ( $8 \mathrm{~Hz}-1.6 \mathrm{kHz}$ ), sweep speed (5 msec/division), gain $(10 \mathrm{uV})$, stimulation intensity (208 V), and duration (0.05 msec).

Measurements of distance, response latencies, and amplitude were carried out in a standard fashion using onset latencies, and base line to peak amplitude. Measurements of peak-peak amplitudes were used for sensory responses [15]. Sensory nerve conduction. The sensory nerve action potential (SNAP) of the median nerve was measured by the antidromic technique on the index finger with ring electrodes placed $2.5 \mathrm{~cm}$ apart. The fixed distal distance for sensory NCV was $20 \mathrm{~mm}$ proximal to the distal wrist crease and for ulnar was measured by antidromic technique on the little finger. The sural SNAPs were recorded behind the lateral malleolus on the posterior lateral aspect of the leg, $14 \mathrm{~cm}$ from the active electrode with a fixed distal distance of around $140 \mathrm{~mm}$ above the recording electrode [16]. Sensory nerve action potential latency, amplitude and conduction velocity were measured. Motor nerves conduction. The compound muscle action potentials (MNAP) of the median were obtained using supramaximal stimulation of the nerves at the wrist and elbow, with the distal distance for motor NCV $80 \mathrm{~mm}$ above the recording electrode and for ulnar nerve was done at wrist and elbow [15].

For common peroneal conduction velocity, the nerve was stimulated at the anterior surface of the ankle, and at the popliteal fossa. Motor nerve action potential latency, amplitude and conduction velocity were measured, also for tibial nerve was done behind medial malleolus. To reduce occurrence of bias, scoring of peripheral neuropathies was performed by the same investigator prior to accessing any sort of data that gives information on vit $\mathrm{D}$ status. Serum samples were sent for analysis after completing data extraction and peripheral neuropathy diagnosis.

Statistical Analysis: Statistical analyses were performed using the Statistical Package for Social Sciences SPSS software, version 19.0 (SPSS, Inc., Chicago, IL, USA) by an expert statistician. All data were reported as mean \pm SD. Two-way ANOVA was performed to compare between the study groups. A difference of $\mathrm{p}<0.05$ was considered significant and confidence interval of 95\%. Post ANOVA test analysis including Tukey-Kramer multiple comparisons test was used for comparison between the different study groups. Spearman rho correlation coefficient was applied to find the strength of correlation between continuous quantitative variables and neuropathy parameters. A difference of $p<0.05$ was considered significant. In addition, Chi squared analysis including linear regression analysis was used to examine the relationship between neuropathy measures including median and common peroneal nerves motor potential latency and motor conduction velocity also median and sural sensory conduction velocity, sural nerve potential amplitude all as (dependent variables), with DPN potential risk factors as vit D level, $\mathrm{HbA1c}, \mathrm{BMI}$, and atherogenic index as (independent variables). A 5\% level was chosen as a level of significance in all statistical significance tests used.

\section{Results}

Table 1 summarizes the cross relation between DPN group III and Vit D status, compared to those and with early neuropathy group II ,we found that $90 \%(n=27)$ of early DPN patients had normal Vit D status, while only 10\% (5) of patients with late DPN have normal Vit D status. Only $10 \%(\mathrm{n}=3)$ of early DPN patients have Vit D deficiency, while $90 \%(\mathrm{~N}=45)$ of patients with late DPN have deficient Vit D status. Table 2 summarize the characteristics of DPN group(III) compared to diabetics without DPN group I, and 
early DPN group (II) in relation to Vit D status and demographic data. Subjects with late DPN had deficient Vit D status compared to normal Vit D status in group I and early DPN group II ( $p=0.001$ * and $\mathrm{p}=0.002^{*}$ ), DPN group( III) had significantly longer diabetes duration $\left(\mathrm{p}<0.002^{*}\right.$ and $\left.\mathrm{p}<0.001^{*}\right)$ and had a significantly higher mean HgbA1c $\left(\mathrm{p}<0.000^{*}\right.$ and $\left.\mathrm{p}<0.001^{*}\right)$ and significantly high atherogenic index(AI) $\left(\mathrm{p}<0.003^{*}\right.$ and $\left.\mathrm{p}<0.002^{*}\right)$ all compared to group I and early DPN group II , and there was significant change between group III compared to group I and II in relation to BMI $\left(\mathrm{p}<0.005^{*}\right.$ and $\left.\mathrm{p}<0.012^{*}\right)$.

Table 1: Cross relation between Vit D deficiency status and Diabetic Peripheral Neuropathy (DPN).

\begin{tabular}{|c|c|c|c|}
\hline \multirow{2}{*}{ Vit D status } & \multicolumn{3}{|c|}{ Diabetic Peripheral Neuropathy (DPN) } \\
\cline { 2 - 4 } & Early DPN Group II $\mathbf{n = 3 0}$ & Late DPN Group III n=50 & Total n=80 \\
\hline -Normal & $27(90 \%)$ & $5(10 \%)$ & 32 \\
\hline -Deficiency & $3(10 \%)$ & $45(90 \%)$ & 48 \\
\hline Total & 30 & 50 & 80 \\
\hline
\end{tabular}

Table 2: The characteristics of DPN group (III) compared to Group I and possible DPN group (II) in relation to Vit D status and demographic data.

\begin{tabular}{|c|c|c|c|c|}
\hline Variable & $\begin{array}{l}\text { Diabetic Patients without } \\
\text { DPN Group I N (15) }\end{array}$ & $\begin{array}{l}\text { Diabetic Patients without } \\
\text { DPN Group I N (15) }\end{array}$ & $\begin{array}{l}\text { Diabetic Patients without } \\
\text { DPN Group I N (15) }\end{array}$ & P-Value \\
\hline Vit D level (25-OH-Vit D (ng/ml) & $21 \pm 0.01$ & $22 \pm 1.04$ & $15 \pm 0.32$ & $\begin{array}{c}0.002^{*} \\
0.001 ¥ \\
0.07\end{array}$ \\
\hline Type II DM duration (years) & $5 \pm 0.002$ & $9 \pm 0.1$ & $12 \pm 1.2$ & $\begin{array}{c}0.061 \\
0.002 ¥ \\
0.000 \AA\end{array}$ \\
\hline $\mathrm{HbA1c}(\mathrm{nmol} / \mathrm{ml})$ & $5.2 \pm 1.1$ & $7.3 \pm 1.2$ & $9.41 \pm 0.5$ & $\begin{array}{c}0.001^{*} \\
0.000 ¥ \\
0.001 \AA\end{array}$ \\
\hline $\mathrm{BMI} \mathrm{kg} / \mathrm{m}^{2}$ & $24.2 \pm 0.9$ & $25.1 \pm 5.3$ & $29.0 \pm 5.5$ & $\begin{array}{c}0.012^{*} \\
0.005 ¥ \\
0.09\end{array}$ \\
\hline Atherogenic Index (AI) & $21 \pm 1.01$ & & & $\begin{array}{c}0.002^{*} \\
0.003 ¥ \\
0.1\end{array}$ \\
\hline
\end{tabular}

Note: Results are expressed as mean \pm standard deviation.

*Significant changes Group III compared with Group II $(\mathrm{p}<0.05)$.

$¥$ Significant changes Group III compared with control Group I $(\mathrm{p}<0.05)$.

( Significant changes Group II compared with control Group I $(\mathrm{p}<0.05)$.

There was a significant increase between group I and early DPN group II in relation to diabetes duration $\left(\mathrm{p}=0.000^{*}\right)$ and in HgbA1c $\left(\mathrm{p}<0.001^{*}\right)$, but other parametes showed insignificant change e.g in relation to Vit D status $(\mathrm{p}<0.07)$ and for BMI $(\mathrm{p}<0.09)$,also for $\operatorname{AI}(p<0.1)$. The comparison of DPN group III with Group I and early DPN group(1I) in relation to nerve conduction studies(NCSs) showed that there is significant prolongation of motor nerve action potential latency of median, ulnar, common peroneal and tibial nerves in Group III compared to group II $\left(\mathrm{p}<0.012^{*}, \mathrm{p}<0.001^{*}\right.$, $\mathrm{p}<0.032^{*}$ and $\mathrm{p}<0.016^{*}$ ), Also with group I $\left(\mathrm{p}<0.011^{*}, \mathrm{p}<0.001^{*}\right.$, $\mathrm{p}<0.02^{*}$ and $\mathrm{p}<0.09^{*}$ ), Also there was significantly reduced motor conduction velocity of median, ulnar, common peroneal and tibial nerves in Group III compared to group II $\left(\mathrm{p}<0.025^{*}, \mathrm{p}<0.044^{*}\right.$, $\mathrm{p}<0.032^{*}$ and $\left.\mathrm{p}<0.001^{*}\right)$. Also with group I $\left(\mathrm{p}<0.022^{*}, \mathrm{p}<0.033^{*}\right.$, $\mathrm{p}<0.012^{*}$ and $\mathrm{p}<0.001^{*}$ ). Concerning the sensory nerve conduction studies there was significant prolongation of sensory nerve action potential latency of median,ulnar,and sural nerves in Group III compared to group II $\left(\mathrm{p}<0.019^{*}, \mathrm{p}<0.015^{*}\right.$ and $\left.\mathrm{p}<0.033^{*}\right)$, Also with group I ( $\left.p<0.001^{*}, \mathrm{p}<0.003^{*}, \mathrm{p}<0.02^{*}\right)$.

Also, there was significantly reduced sensory conduction velocity of median,ulnar and sural nerves in Group III compared to group II ( $p<0.002^{*}, \mathrm{p}<0.007^{*}$ and $\left.\mathrm{p}<0.003^{*}\right)$, also with group I ( $\mathrm{p}<0.012^{*}$, $\mathrm{p}<0.003^{*}$ and $\mathrm{p}<0.022^{*}$ ). Concerning sural nerve potential amplitude (Samp) it was significantly reduced in Group III of DPN compared to group II of possible DPN ( $\left.<<0.003^{*}\right)$, also with Group I 
$\left(\mathrm{p}<0.005^{*}\right.$ ) (Table 3). Comparison of group II to group I showed a significant prolongation of sensory sural nerve action potential latency $\left(\mathrm{p}<0.01^{*}\right)$ and a significant decrease of sural nerve potential amplitude (Samp) $\left(\mathrm{p}<0.001^{*}\right)$. The relationship between risk factors and individual neuropathy characteristics was examined for those with DPN. Table IV represents DPN measures by electrophysiological examination in Group II (DPN) correlated with potential risk factors of DPN as diabetes duration, BMI, HbA1c, atherogenic index and Vit D status. Median nerve motor action potential latency as well as motor conduction velocity which directly reflects large myelinated motor fiber integrity showed that the latency significantly correlated positively with BMI ,Hb A1c and atherogenic in$\operatorname{dex}(\mathrm{r}=0.520, \mathrm{r}=0.409$ and $\mathrm{r}=0.661)\left(\mathrm{p}<0.002^{*}\right),\left(\mathrm{p}<0.002^{*}\right)$ and $(\mathrm{p}$ $<0.022^{*}$ ) respectively, but there was a significant negative strong correlation with Vit D levels but not with diabetes duration, $(\mathrm{r}$ $=-0.721$ and $\mathrm{r}=0.175)\left(\mathrm{p}<0.001^{*}\right)$ and $(\mathrm{p}<0.052)$ respectively.

Table 3: The characteristics of DPN group (III) compared to Group (I) and Possible DPN group (II) in relation to Nerve Conduction Studies (NCSs).

\begin{tabular}{|c|c|c|c|c|c|c|c|c|}
\hline \multirow{2}{*}{ 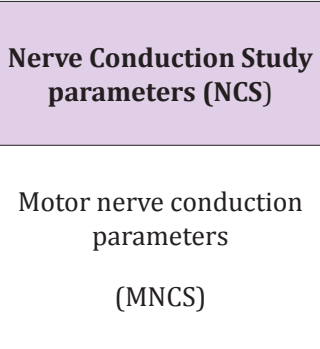 } & \multicolumn{2}{|r|}{$\begin{array}{c}\text { GroupI } \\
\text { (15) }\end{array}$} & \multicolumn{2}{|c|}{$\begin{array}{c}\text { Early DPN } \\
\text { Group II } \\
\text { n (30) }\end{array}$} & \multicolumn{2}{|c|}{$\begin{array}{c}\text { Late DPN } \\
\text { Group III } \\
\text { n (50) }\end{array}$} & \multirow{2}{*}{$\begin{array}{l}\text { P-Value } \\
\\
\text { (latency) } \\
\text { (msec) }\end{array}$} & \multirow{2}{*}{$\begin{array}{c}\text { P-Value } \\
\text { (CV) }\end{array}$} \\
\hline & $\begin{array}{l}\text { Latency } \\
\text { (msec) }\end{array}$ & $\begin{array}{l}\text { Conduction velocity } \\
\qquad(\mathrm{m} / \mathrm{sec})\end{array}$ & $\begin{array}{l}\text { Latency } \\
\text { (msec) }\end{array}$ & $\begin{array}{c}\text { Conduction } \\
\text { velocity } \\
\text { (CV } \\
\mathrm{m} / \mathrm{sec} \text { ) }\end{array}$ & $\begin{array}{l}\text { Latency } \\
\text { (msec) }\end{array}$ & $\begin{array}{l}\text { Conduction } \\
\text { velocity } \\
(\mathrm{CV} \mathrm{m} / \mathrm{sec})\end{array}$ & & \\
\hline 1-Median nerve & $3.0 \pm 0.1$ & $51 \pm 1.0$ & $3.1 \pm 0.31$ & $51 \pm 1.01$ & $4.7 \pm 0.13$ & $421 \pm 1.03$ & $\begin{array}{l}0.012^{*} \\
0.011 ¥\end{array}$ & $\begin{array}{l}0.025^{*} \\
0.022 ¥\end{array}$ \\
\hline 2-Ulnar nerve & $3 \pm 0.12$ & $50 \pm 1.13$ & $3.2 \pm 0.12$ & $53 \pm 1.03$ & $4.2 \pm 0.002$ & $45.4 \pm 0.14$ & $\begin{array}{l}0.001^{*} \\
0.001 ¥\end{array}$ & $\begin{array}{l}0.044^{*} \\
0.033 ¥\end{array}$ \\
\hline 3-Peroneal nerve & $5.1 \pm 0.11$ & $40 \pm 1.33$ & $5.3 \pm 0.13$ & $42 \pm 1.13$ & $7.45 \pm 1.33$ & $39.23 \pm 123$ & $\begin{array}{c}0.032^{*} \\
0.02 ¥\end{array}$ & $\begin{array}{l}0.032 * \\
0.012 ¥\end{array}$ \\
\hline 4-Tibial nerve & $5.1 \pm 0.22$ & $42.01 \pm 0.01$ & $5.5 \pm 0.24$ & $43.03 \pm 0.6$ & $7.81 \pm 0.45$ & $32.01 \pm 2.11$ & $\begin{array}{l}0.016^{*} \\
0.09 ¥\end{array}$ & $\begin{array}{l}0.001 * \\
0.001 ¥\end{array}$ \\
\hline \multicolumn{9}{|c|}{ Sensory nerve conduction parameters (SNCS) } \\
\hline 1-Median nerve & $3 \pm 0.11$ & $38.1 \pm 1.01$ & $3.1 \pm 0.01$ & $39.1 \pm 2.01$ & $4.1 \pm 0.33$ & $31.3 \pm 0.32$ & $\begin{array}{l}0.019^{*} \\
0.001 ¥\end{array}$ & $\begin{array}{l}0.002 * \\
0.012 ¥\end{array}$ \\
\hline 2-Ulnar nerve & $3.0 \pm 0.02$ & $39 \pm 1.33$ & $3.3 \pm 0.02$ & $39.6 \pm 1.43$ & 3.9.11 \pm 0.002 & $34.4 \pm 0.67$ & $\begin{array}{l}0.015^{*} \\
0.003 ¥\end{array}$ & $\begin{array}{l}0.007 * \\
0.003 ¥\end{array}$ \\
\hline 3-Sural nerve & $3.3 \pm 0.11$ & $38.13 \pm 1.53$ & $4.0 \pm 0.13$ & $39.43 \pm 1.53$ & $4.98 \pm 1.34$ & $28.06 \pm 0.33$ & $\begin{array}{l}0.033^{*} \\
0.02 ¥ \\
0.01 \circledast\end{array}$ & $\begin{array}{l}0.003^{*} \\
0.022 ¥\end{array}$ \\
\hline $\begin{array}{l}\text { 4- Sural nerve sensory } \\
\text { amplitude } \\
\text { Samp (Mv) }\end{array}$ & & $5.5 \pm 0.23$ & & 0.33 & 3.5 & & $\begin{array}{l}0.0 \\
0.00 \\
0.00\end{array}$ & \\
\hline
\end{tabular}


Note: Results are expressed as mean \pm standard deviation.

*Significant changes Group III compared with Group II $(p<0.05)$.

$¥$ Significant changes Group III compared with control Group I $(\mathrm{p}<0.05)$.

( Significant changes Group I Icompared with control Group I $(\mathrm{p}<0.05)$.

Whereas the median motor conduction velocity is significantly correlated negatively with BMI , Hb A1c and atherogenic index ( $\mathrm{r}$ $=-0.521, \mathrm{r}=-0.556$ and $\mathrm{r}=-0.533)\left(\mathrm{p}<0.001^{*}\right),\left(\mathrm{p}<0.000^{*}\right)$ and $(\mathrm{p}$ $<0.001^{*}$ ) respectively and positively correlated significantly with Vit $D$ status but not with diabetes duration ( $r=0.889$ and $r=0.209)$ $\left(p<0.007^{*}\right)$ and $(p<0.062)$ respectively. Peroneal motor conduction velocity which represent a large myelinated motor fiber surrogate correlated significantly and negatively with BMI , $\mathrm{Hb} \mathrm{A} 1 \mathrm{c}$ and atherogenic index $(r=-0.536, r=-0.405$ and $r=-0.653)\left(p<0.001^{*}\right),(p$ $\left.<0.002^{*}\right)$ and $\left(\mathrm{p}<0.034^{*}\right)$ respectively and positively correlated with Vit D levels but not with diabetes duration( $\mathrm{r}=0.684$, $\mathrm{r}$ $=0.355)\left(p<0.034^{*}\right),(p<0.095)$ respectively. Sural nerve sensory amplitude (Samp) and sensory conduction velocity which reflect large sensory myelinated fiber integrity significantly correlated negatively with BMI ,Hb A1c and atherogenic index, for sural sensory amplitude $(r=-0.537, r=-0564$ and $r=-0.693)\left(p<0.001^{*}\right)$, $(p$ $\left.<0.019^{*}\right),\left(\mathrm{p}<0.001^{*}\right)$ respectively, and for sural sensory conduction velocity $(r=-0.621, r=-0.672$ and $r-=0.623)\left(p<0.001^{*}\right),\left(p<0.012^{*}\right)$ and $\left(\mathrm{p}<0.010^{*}\right)$ respectively.

Also Sural nerve sensory amplitude and sensory conduction velocity correlated posititively, significantly and strongly with vit D levels and with diabetes duration, for sural sensory amplitude ( $\mathrm{r}$ $=0.943$ and $\mathrm{r}=0.247)\left(\mathrm{p}<0.001^{*}\right)$ and $(\mathrm{p}<0.001)$ respectively, and for sural sensory conduction velocity $(r=0.857, r=0.236)\left(p<0.001^{*}\right)$ and $(\mathrm{p}<0.005)$ respectively. Linear regression analysis was carried out in DPN group III for Vit D level (as an independent variable), and median motor potential latency, median motor conduction velocity, sural nerves sensory conduction velocity, sural Samp and peroneal motor conduction velocity (as dependent variables). The analysis revealed Vit D deficiency status as a predictor of DPN measures as median motor potential latency, median motor conduction velocity , sural nerves sensory conduction velocity, sural Samp and peroneal motor conduction velocity in the neuropathy group $(B=-$ $\left.0.66, p=0.022^{*}\right)\left(\beta=0.313, p=0.026^{*}\right),\left(\beta=-0.65, p=0.001^{*}\right),(ß=-$ $\left.0.76, p=0.002^{*}\right)$ and $\left(ß=0.413, p=0.016^{*}\right)$.

Linear regression analysis was carried out in DPN group III for BMI (as an independent variable), and median motor potential latency, median motor conduction velocity, sural nerves sensory conduction velocity, sural Samp and peroneal motor conduction velocity (as dependent variables). The analysis revealed BMI is as a predictor of sural sensory conduction velocity, sural Samp $\left(\mathrm{p}=0.002^{*}\right)\left(\mathrm{p}=0.001^{*}\right)$. But not median motor potential latency, median motor conduction velocity and peroneal motor latency and conduction velocity in the neuropathy group $(p=0.071),(p=0.062)$ and $(\mathrm{p}=0.066)$. Also linear regression analysis was carried out in DPN group III for HbA1c (as an independent variable), and median motor potential latency, median motor conduction velocity , sural nerves sensory conduction velocity, sural Samp and peroneal motor conduction velocity (as dependent variables).The analysis revealed also that $\mathrm{HbA1c}$ is a predictor of sural sensory conduction velocity , sural Samp $\left(p=0.011^{*}\right)\left(p=0.001^{*}\right)$. Also, for median and peroneal motor conduction velocity in the neuropathy group $\left(\mathrm{p}=0.001^{*}\right)$ and $\left(\mathrm{p}=0.032^{*}\right)$ But not a predictor for median motor potential latency in the neuropathy group $(\mathrm{p}=0.063)$.

Concerning the atherogenic index linear regression analysis was carried out for it (as an independent variable) in DPN group, and median motor potential latency, median motor conduction velocity, sural nerves sensory conduction velocity ,sural Samp and peroneal motor conduction velocity (as dependent variables). The analysis revealed atherogenic index as a predictor of median motor potential latency, median motor conduction velocity , sural nerves sensory conduction velocity, sural Samp and peroneal motor conduction velocity in the neuropathy group $\left(\beta=-0.76, p=0.012^{*}\right)$ $\left(ß=0.413, p=0.023^{*}\right),\left(\beta=-0.65, p=0.001^{*}\right),\left(ß=-0.763, p=0.001^{*}\right)$ and $\left(ß=0.713, p=0.011^{*}\right)$.

\section{Discussion}

The findings of the present study reveals a significant deficiency of Vit D in group III ( late DPN) compared to early DPN (group I) and its negative correlation with DPN electrophysiological measures of median, ulnar, peroneal and tibial nerves motor potential latency , its negative correlation with median and sural nerves sensory potential latency, also its positive correlation with sural nerve sensory amplitude, together with its positive correlation with sensory and motor nerves conduction velocity are in accordance and concistant with the finding of Celil et al. [9] who stated that Vit D deficiency has a possible relationship to diabetic microvascular complications. They postulated that Vitamin D improves end othelial function by reducing vascular inflammation, regulating blood pressure, inhibiting proliferation of vascular smooth muscle cells, and antagonizing the formation of foam cell. Further, antioxidant property of Vitamin D adds to its protective mechanisms on heart and vasculature [9]. Depending on Celil et al. [9] findings we could postulate that vit D deficiency impaires and deteriorate the microvascular circulation to peripheral nerves and initiates all the findings of DPN in the current study.

Rasheed MA et al. [17] stated that altered VitD levels may play a role in the pathogenesis of T2DM and its microvascular complications. Altered expression of Vit D receptors Notch2 and VDR polymorphisms may play a role in the development of microvascular complications as DPN in diabetic patients. These results may assist in early identification and management of diabetic complications may be by vit D supplementation[17]. Vitamin D deficiency is an important risk factor for glucose intolerance and contributes to the etiology and progression of type 
2 diabetes . Recent studies have shown impaired insulin synthesis and secretion in animal models with vitamin D deficiency; diabetes onset can be delayed with 1-25-OH vitamin D intake. Also,1-25- $\mathrm{OH}$ vitamin D concentrations were found to be lower in patients with type 2 diabetes with impaired glucose tolerance than in controls [18]. Vitamin D is a known suppressor of renin biosynthesis, and vitamin D deficiency has been associated with progression of chronic kidney disease (CKD) and diabetic nephropathy. Patients with type 2 diabetes and CKD have an impaired microvascular circulation due to exceptionally high rate of severe $1-25-0 \mathrm{H}$ vitamin D deficiency [18]. These findings could explain the cause for occurrence of DPN in vit D deficiency group.

Wu C et al. [19] showed that vitamin D supplementation was associated with reduced $\mathrm{HbA1c}$ levels among patients with 25-hydroxyvitamin D (25(OH) D) deficiency. Significantly reduced HbA1c levels were also observed in association with vitamin D supplementation in the subgroup including type 2 diabetes patients with a body mass index (BMI) $<30 \mathrm{~kg} \mathrm{~m}-2$.Thus Vit D supplementation could be effective at improving glycemic control in vitamin D deficient or non-obese type 2 diabetes patients.So,it could ameliorate diabetic microvascular complications. Akbari M et al. [20] conducted a study to summarize the effect of vitamin D supplementation on glucose homeostasis parameters and lipid profiles in gestational diabetes (GDM) patients. The results showed that vitamin D supplementation significantly reduced the homeostasis model assessment of insulin resistance (HOMA-IR) and cholesterol levels significantly. They found no beneficial effect of vitamin D supplementation on fasting plasma glucose (FPG), insulin, HbA1c, total-, HDL-cholester$\mathrm{ol}$, and triglycerides concentrations, and this is in contrast to the findings of Wu C et al. [19].

The currentstudy showed thatserum 1-25(OH)D concentrations were significantly lower in subjects with DPN than in those with early possible DPN .Linear regression analysis revealed that serum 25(OH)D deficiency may be a risk factor of DPN among saudi females with type 2 diabetis, with high BMI, a long duration of diabetes and high atherogenic index. These are as the findings of Xiaoyan xiao et al. [7] who studied the relation of vit D deficiency with prevelance of diabetic nephropathy. The challenge is to determine the mechanisms of vitamin $\mathrm{D}$ action in diabetic patients for recommendation of vitamin D supplementation in the future for diabetic patients to that reduce the risks of T2DM and progression to DPN. Vitamin D deficiency is prevalent in patients with diabetic nephropathy and increases in severity with diabetic nephropathy progression. Age, obesity, glucose level and renal function largely affect 25(OH)D deficiency in diabetic nephropathy [21]. These findings support the notion that vit D deficiency deteriorates the microvascular circulation.

In the present study, the relationship between glycemic control, BMI, diabetes duration and atherogenic index with major features of neuropathy was examined in a cohort of female participants with type 2 diabetes to better understand risk factors for early possible neuropathy development versus patients with well-established and more advanced neuropathy. There were significant differences in key measures of neuropathy severity as median, ulnar, tibial and Peroneal motor latency and conduction velocity also sensory median and sural latency and sensory amplitude between diagnostic groups that with possible early DPN and actual DPN group (Table 4). There were significant differences between each of the two diagnostic groups for DPN measures at the arm, foot and distal leg. These findings suggest that both sensory and motor large myelinated nerve fibers are affected in the course of neuropathy. The correlation data suggests there may be differential impact of diabetic duration, dyslipidemia, obesity and hyperglycemia on individual neuropathy measures.

Table 4: DPN measures in group III by electrophysiological examination correlated with diabetes duration, BMI, HbA1c, atherogenic index and with Vit D status as different risk factors of DPN.

\begin{tabular}{|c|c|c|c|c|c|c|}
\hline Measure & & Diabetes duration & BMI & HbA1c & Atherogenic index & Vit D status \\
\hline \multirow{2}{*}{-Median motor latency } & Correlation coefficient ${ }^{\circledR}$ & 0.175 & 0.52 & 0.409 & 0.661 & -0.721 \\
\hline & $P$ value & $\mathrm{p}<0.052$ & $\mathrm{p}<0.002^{*}$ & $\mathrm{p}<0.002^{*}$ & $\mathrm{p}<0.022^{*}$ & $\mathrm{p}<0.001^{*}$ \\
\hline \multirow[t]{2}{*}{$\begin{array}{l}\text {-Median motor conduction } \\
\text { velocity }\end{array}$} & Correlation coefficient ${ }^{\circledR}$ & 0.209 & -0.521 & -0.556 & -0.533 & 0.889 \\
\hline & $P$ value & $\mathrm{p}<0.062$ & $\mathrm{p}<0.001^{*}$ & $\mathrm{p}<0.000^{*}$ & $\mathrm{p}<0.001^{*}$ & $\mathrm{p}<0.007^{*}$ \\
\hline $\begin{array}{c}\text {-Sural sensory amplitude } \\
\text { Samp }\end{array}$ & Correlation coefficient ${ }^{\circledR}$ & 0.247 & -0.537 & -0.564 & -0.693 & 0.943 \\
\hline & $P$ value & $\mathrm{p}<0.001^{*}$ & $\mathrm{p}<0.001^{*}$ & $\mathrm{p}<0.019^{*}$ & $\mathrm{p}<0.001^{*}$ & $\mathrm{p}<0.001^{*}$ \\
\hline
\end{tabular}




\begin{tabular}{|c|c|c|c|c|c|c|}
\hline \multirow{2}{*}{$\begin{array}{l}\text {-Sural sensory conduction } \\
\text { velocity }\end{array}$} & Correlation coefficient ${ }^{\circledR}$ & 0.236 & -0.621 & -0.672 & -0.623 & 0.857 \\
\hline & $P$ value & $\mathrm{p}<0.005^{*}$ & $\mathrm{p}<0.001^{*}$ & $\mathrm{p}<0.012^{*}$ & $\mathrm{p}<0.010^{*}$ & $\mathrm{p}<0.001^{*}$ \\
\hline \multirow[t]{2}{*}{$\begin{array}{l}\text {-Peroneal motor conduction } \\
\text { velocity }\end{array}$} & Correlation coefficient ${ }^{\circledR}$ & 0.355 & -0.536 & -0.405 & -0.653 & 0.684 \\
\hline & $P$ value & $\mathrm{p}<0.095$ & $\mathrm{p}<0.001 *$ & $\mathrm{p}<0.002^{*}$ & $\mathrm{p}<0.034 *$ & $\mathrm{p}<0.034^{*}$ \\
\hline
\end{tabular}

Note: Correlation coefficients and $\mathrm{p}$ values are displayed for different relationships.

*Significant correlation. $\left(\mathrm{p}<0.05^{*}\right)$.

The results of the current study indicate that bad glycemic control, obesity and hyper dyslipidemia are significant early diabetic neuropathy risk factors, Indeed,hyperlipidemia, glucose intolerence and obesity significantly correlated with large nerve fiber integrity ,and are more closely correlated with large myelinated fiber function as shown by nerve potential latency and conduction velocity. These findings suggest disparate pathogenesis for injury to motor and sensory myelinated axons and offer additional support to a competing narrative to the traditional understanding of diabetic neuropathy as driven only by prolonged sustained hyperglycemia. Indeed, results of the current study suggesting toxic adiposity, bad glucemic control and dyslipidemia as indexed by high atherogenic index may be more important contributors to diabetic neuropathy.

The findings of the present study are consistant with the study of Fröhlich-Reiterer et al. [22] who mentioned that diabetes-related microvascular and macrovascular complications, as retinopathy, nephropathy and neuropathy are life-threatening complications in children and adolescents with type 1 diabetes mellitus (T1DM). They stated that risk factors for the development of complications are longer duration of diabetes, older age and puberty. Further risk factors include smoking, hypertension, higher body mass index and dyslipoproteinaemia. They confirmed that targetlevels to reduce the risk of microvascular and macrovascular complications in children and adolescents with T1DM are the following: HbA1c $<7.5 \%$, lipids in normal range, blood pressure $<90$ th percentile by age, sex and height, $\mathrm{BMI}<95$ th percentile, no smoking and physical activity.

The current study stated that high BMI is a risk factor for DPN., this is in accordance to the findings of Herman et al. [23] study who compared obese and thin subjects, they have shown relative abnormalities of both nerve conduction measures and small fiber axonal function in the obese subjects, most of who were asymptomatic. The findings of the current study are in contrast to the findings in animal models of diet-induced obesity which demonstrated both microvascular and neural dysfunction in nonhyperglycemic animals [24]. These mice develop neuropathy in the absence of hyperglycemia but in presence of hyperlipidemia [25] Non-diabetic mice fed a high-fat diet develop increased levels of oxidized low density lipoproteins, free fatty acids and triglycerides, as well as evidence of increased systemic and nerve oxidative stress. These mice develop neuropathy in the absence of hyperglycemia [25]. Results of the current study adds substantially to this evolving literature suggesting toxic adiposity and dyslipidemia may be more important contributors to diabetic and idiopathic neuropathy risk than has previously been recognized [25]. Correlation data of the current study assessed the relationship.

Also, previous studies stated that higher BMI is an independent predictor of major renal events in patients with type 2 diabetes. These findings encourage weight loss to improve nephroprotection in these patients [26,27]. With reference to these findings we can state that high BMI is an independent predictor of DPN. The findings of the current study that high atherogenic index is a predictor of DPN are in line with study of Obrosova et al. [28] who stated that non-diabetic mice fed a high-fat diet develop increased levels of oxidized low density lipoproteins, free fatty acids and triglycerides, as well as evidence of increased systemic and nerve oxidative stress all are initiators of neuronal damage. Thus, nerve oxidative stress with the high atherogenic index are important causative factors in initiation of DPN in the current study. These results of the present study indicate that obesity and hypertriglyceridemia are significant diabetic neuropathy risk factors. Moreover, hypertriglyceridemia and obesity significantly correlated with large motor and sensory myelinated dysfunction.

Previous studies considered atherogenic dyslipidaemia as an important and modifiable contributor to the lipid related residual cardiovascular risk. Atherogenic dyslipidaemia is defined as an imbalance between proatherogenic triglycerides-rich apoBcontaining lipoproteins and antiatherogenic index containing lipoproteins [29]. The current study showed that subjects who had DPN had a longer duration of diabetes, and higher mean hemoglobin A1c than those who have possible early neuropathy, also glucose control more closely correlated with large myelinated fiber function (NCS conduction velocity). These findings suggest disparate pathogenesis for injury to motor and sensory axons and myelinated motor fibers, and offer additional support to a competing narrative 
to the traditional understanding of diabetic neuropathy as driven by prolonged sustained hyperglycemia as shown in previous studies [29], Dyck et al. [30] stated that motor conduction velocity is an appealing surrogate measure because it declines early in the course of diabetic neuropathy and it is responsive to aggressive glycemic control in type 1 diabetes, and is reproducible. Their correlation data assess the relationship between potential risk factors and disease severity. Large myelinated nerve fiber measures such as motor conduction velocity or sensory nerve action potential amplitude correlated best with hemoglobin A1c and height, both of which are well-recognized neuropathy risk factors [30].

Sorop o et al. [31] stated that vascular stiffness, vasoconstriction by endothelin-1 and Coronary microvascular dysfunction are enhanced after long-term diabetes, high HbA1c, and hypercholesterolemia. Good glycemic control is essential in preventing diabetic Complications. A persistent effect of high HbA1c on diabetic complications are strong [31,32]. Interestingly, sural sensory amplitude and conduction velocity which measures sensory large myelinated nerve integrity also correlated with BMI and atherogenic index.The findings of this study suggest hyperglycemia, Hb A1c, obesity and dyslipidemia may have distinct but overlapping effects on peripheral sensory nerve function. This fact suggests these risk factors may be particularly important in loss of sensory axons which is the potential pathology underlying patient symptoms and disability. Also, hyperglycemia, dyslipidemia and obesity appear to cause preferential injury to large myelinated motor fibers, reflected by slowing of motor nerve conduction velocity and prolongation of motor nerves latencies in the present study.

\section{Study Limitations}

The number of patients wishing to participate was too low for a random selection. There was high rates of refusal and dropout from groups. Some patients attended for blood collection without fasting, and some patients refused blood collection by venipuncture because of fear and distress of needles, and hence were excluded from the study. There were also a few patients with other diabetic complications together with neuropathy, and so they were also excluded from the study.

\section{Conclusion}

The cost of treating diabetes patients constitutes a large part of the total health care budget, and it is thus important from the economic perspective to have a sound knowledge of the effects of glycemic control, body weight, lipid profile, and Vit D status as different risk factors on diabetic microvascular complications. In conclusion, our findings suggested Vit D deficiency is an independent risk factor of DPN in T2DM patients. T2DM patients with Vit D deficiency had severer neurological symptoms and signs, and lower NCV of most nerves compared with normal Vit D counterparts. More attention should be paid for early screening for signs and symptoms of DPN which may be necessary in T2DM patients with Vit D deficiency. The likelihood of having neuropathy increased as the number of risk factors present increased. There is an urgent need for better surrogate markers of early axon loss. The observation that there is differential relationship between large sensory and motor fibers lesions measures and hyperglycemia, dyslipidemia marked by high atherogenic index and obesity supports development of potential new endpoint measures. large sensory and motor fiber measures may be particularly important in any study examining the effect of a treatment designed to improve Vit D status, lower lipids , HbA1c and body weight or ameliorate the deleterious effects of central adiposity. Longitudinal studies are required to evaluate the extent to which these risk factors predict future neuropathy development. Our findings also encourage weight loss, good Vit D supplements, lower blood lipids to improve neuroprotection in DPN patients.

\section{Acknowledgment}

The author would like to express gratitude to the College of medicine Research Center, Deanship of Scientific Research, King Saud University, Riyadh, Saudi Arabia for sponsoring and supporting this study. The author also thanks the Diabetic Center of King Abdulaziz University Hospital, King Saud University for their help in recruitment of patients. Thanks, are extended also to the laboratory technicians of the Clinical Physiology Department, King Abdulaziz University Hospital, King Saud University for their help in the electrophysiological measurements

\section{Competing Interest}

None of the authors have professional, personal of financial conflicts of interest to report.

\section{References}

1. Faten A Zakareia (2008) Electrophysiological changes, plasma vascular endothelial growth factor, fatty acid synthase, and adhesion molecules in diabetic neuropathy. Neurosciences 13(4): 374-379.

2. Smith DG, Assal M, Reiber GE, Vath C, Le Master J (2003) Minor environmental trauma and lower extremity amputation in highrisk patients with diabetes: incidence, pivotal events, etiology, and amputation level in a prospectively followed cohort. Foot Ankle Int 24(9): 690-695.

3. Richards-George P Vasculopathy (2001) in the diabetic foot. West Indian Med J 50 (Suppl 1): S18-S20.

4. Antoniades C, Tousoulis D, Tountas C, Tentolouris C, ToutouzaM, et al. (2004) Vascular endothelium and inflammatory process, in patients with combined Type 2 diabetes mellitus and coronary atherosclerosis: The effects of vitamin C. Diabet Med 21(6): 552-558.

5. Ferrara N, Davis-Smyth T (1997) The biology of vascular endothelial growth factor. Endocr Rev 18(1): 4-25.

6. Seckin D, Ilhan N, Ilhan N, Ertugrul S (2006) Glycaemic control, markers of endothelial cell activation and oxidative stress in children with type 1 diabetes mellitus. Diabetes Res Clin Pract 73(2): 191-197.

7. Xiaoyan Xiao, Yajuan Wang, Yanlian Hou, Feng Han, Jianmin Ren, et al. (2016) Vitamin D deficiency and related risk factors in patients with diabetic nephropathy. Journal of International Medical Research 44(3): 673-684.

8. Sylvia Christakos, Puneet Dhawan, Annemieke Verstuyf, Lieve Verlinden, Geert Carmeliet (2016) Vitamin D: Metabolism, molecular mechanism of action, and pleiotropic effects. Physiol Rev 96(1): 365-408. 
9. Celil Alper Usluogullari, Fevzi Balkan, Sedat Caner, Rifki Ucler, Cafer Kaya, et al. (2015) The relationship between microvascular complications and vitamin D deficiency in type 2 diabetes mellitus. BMC Endocrine Disorders 15: 33

10. Nishanthi Anandabaskar, Sandhiya Selvarajan, Steven Aibor Dkhar Sadish Kumar Kamalanathan, Kadhiravan Tamilarasu, et al. (2017) Effect of vitamin d supplementation on vascular functions and oxidative stress in type 2 diabetic patients with vitamin d deficiency. Indian J Endocrinol Metab 21(4): 555-563.

11. DM Li, Zhang Y, Ding B, Liu BL, Jiang LL, et al. (2013) The association between vitamin $\mathrm{D}$ deficiency and diabetic nephropathy in type 2 diabetic patients. Zhonghua Nei Ke Za Zhi 52(11): 970-974.

12. Guerin M, Legoff W, Lassel TS, VanTol A, Steiner G, et al. (2001) Atherogenic role of elevated CE transfer from HDL to VLDL and dense LDL in type 2 diabetics. Arterioscler Thromb Vasc Biol 21(2): 282-288.

13. Manninen V, Tenkanen L, Koshinen P, Huttunen JK, Mänttäri M, et al. (2002) Joint effects of serum triglyceride and LDL cholesterol and HDL cholesterol concentrations on coronary heart disease risk in the Helsinki Heart Study: Implications for treatment. Circulation 85(1): 37-45.

14. Huang M, LaLuzerne P, Winters D, Sullivan D (2009) Measurement of vitamin $\mathrm{D}$ in foods and nutritional supplements by liquid chromatography/tandem mass spectrometry. J AOAC Int 92(5): 1327 1335.

15. Kimura J (1984) Principles and pitfalls of nerve conduction studies. Ann Neurol 16: 415-429.

16. Bril V, Buchanan RA (2004) Aldose reductase inhibition by AS-3201 in sural nerve from patients with diabetic sensorimotor polyneuropathy. Diabetes Care 27(10): 2369-2375

17. Rasheed MA, Kantoush N, Abd El-Ghaffar N, Farouk H, Kamel S, et al. (2017) Expression of JAZF1, ABCC8, KCNJ11and Notch2 genes and vitamin $\mathrm{D}$ receptor polymorphisms in type 2 diabetes, and their association with microvascular complications. Ther Adv Endocrinol Metab 8(6): 97-108.

18. Park S, Kim DS, Kang S (2016). Vitamin D deficiency impairs glucosestimulated insulin secretion and increases insulin resistance by reducing PPAR- $\gamma$ expression in nonobese Type 2 diabetic rats.J Nutr Biochem 27 257-265.

19. Wu C, Qiu S, Zhu X, Li L. (2017) Vitamin D supplementation and glycemic control in type 2 diabetes patients: A systematic review and metaanalysis. Metabolism 73: 67-76.

20. Akbari M, Moosazadeh M, Lankarani KB, Tabrizi R, Samimi M, et al (2017) Effect of vitamin d supplementation on glucose metabolism and lipid profiles in patients with gestational diabetes: A systematic review

\section{ISSN: 2574-1241}

\section{DOI: $10.26717 /$ BJSTR.2019.20.003460}

Faten abulhady zakareia. Biomed J Sci \& Tech Res

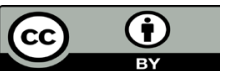

This work is licensed under Creative

Commons Attribution 4.0 License

Submission Link: https://biomedres.us/submit-manuscript.php and meta-analysis of randomized controlled trials. Horm Metab Res 49(9): e3.

21. Sánchez-Hernández RM, García-Cantón C, Lorenzo DL, Quevedo V, Bosch E, et al. (2015) The specific relationship between vitamin D deficiency and diabetic nephropathy among patients with advanced chronic kidney disease: a cross-sectional study in Gran Canaria. Clin Nephrol 83(4): 218-224.

22. Fröhlich-Reiterer EE, Borkenstein MH (2010) Microvascular and macrovascular complications in children and adolescents with type 1 diabetes mellitus. Wien Med Wochenschr 160(15,16): 414-418.

23. Herman RM, Brower JB, Stoddard DG, Casano AR, Targovnik JH, et al. (2007) Prevalence of somatic small fiber neuropathy in obesity. Int J Obes 31(2): 226-235

24. Oltman CL, Coppey LJ, Gellett JS, Davidson EP, Lund DD, et al. (2005) Progression of vascular and neural dysfunction in sciatic nerves of Zucker diabetic fatty and Zucker rats. Am J Physiol Endocrinol Metab. 289(1): E113-122.

25. VincentAM, Hinder LM, Pop-Busui R, Feldman EL (2009) Hyperlipidemia: A new therapeutic target for diabetic neuropathy. J Peripher Nerv Syst 14(4): 257-267.

26. Mohammedi K, Chalmers J, Herrington W, Li Q, Mancia G, et al. (2018) Associations between body mass index and the risk of renal events in patients with type 2 diabetes. Nutr Diabetes 8(1): 7.

27. Millán Núñez-Cortés J, Pedro-Botet Montoya J, Pintó Sala X (2014) Atherogenic dyslipidemia and residual risk .Clin Investig Arterioscler 26(6): 287-292.

28. Obrosova IG, Ilnytska O, Lyzogubov VV, Pavlov IA, Mashtalir N, et al. (2007) High-fat diet induced neuropathy of pre-diabetes and obesity: Effects of healthy" diet and aldose reductase inhibition. Diabetes 56(10): 2598-2608.

29. Callaghan BC, Hur J, Feldman EL (2012) Diabetic neuropathy: One disease or two? Current Opinion in Neurology 25(5): 536-541.

30. Dyck PJ, Norell JE, Tritschler H, Schuette K, Samigullin R, et al. (2007) Challenges in design of multicenter trials: End points assessed longitudinally for change and monotonicity. Diabetes Care 30(10): 2619-2625.

31. Sorop 0, van den Heuvel M, van Ditzhuijzen NS, de Beer VI, Heinonen I, et al. (2016) Coronary microvascular dysfunction after long-term diabetes and hypercholesterolemia. Am J Physiol Heart Circ Physiol 311(6): H1339-H1351.

32. Lind M, Odén A, Fahlén M, Eliasson B (2009) The True value of HbA1c as a predictor of diabetic complications: Simulations of HbA1c variables PloS ONE 4( 2): e4412.

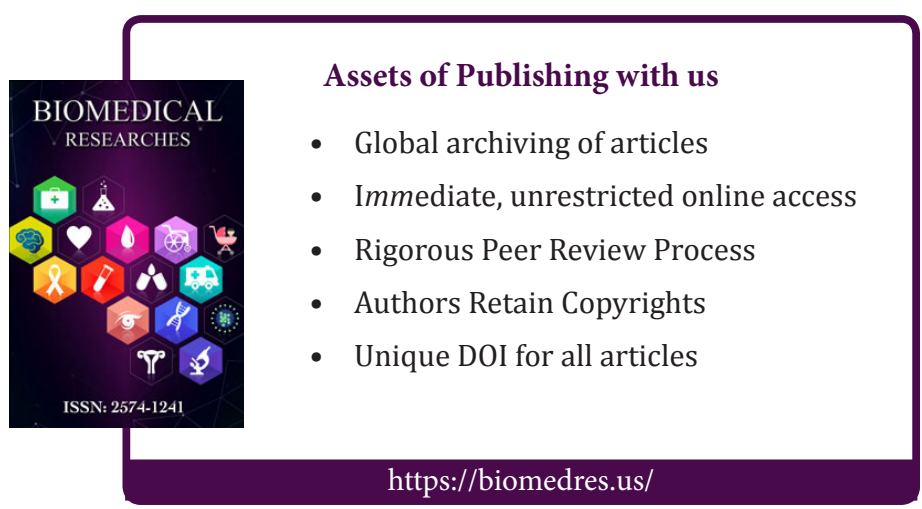

Copyright@ Faten abulhady zakareia | Biomed J Sci \& Tech Res| BJSTR. MS.ID.003460. 